\title{
Evaluación del color, las propiedades texturales y sensoriales de salchicha elaborada con carne de babilla (Caiman Crocodilus Fuscus)
}

\author{
Evaluation of the color, texture and sensory \\ properties of sausage made with spectacled \\ caiman meat (Caiman Crocodilus Fuscus)
}

\begin{abstract}
In the present investigation, 10 tails from spectacled caimans (Caiman crocodilus) $2 \mathrm{Kg}$ each, packed under vacuum at $-20^{\circ} \mathrm{C}$ and stored for 8 days were used. A sausage was made with the lean meat and was evaluated by color, texture and sensory quality to observe freshness, conduct a sensory analysis and texture profile analysis (TPA). The color parameters were $L^{*}=65.04, a^{*}=-0.98$ and $b^{*}=0.98$. Because of the high brightness it is considered a type of white meat. A cut strength measurement of $7.67 \mathrm{Kgf}$ was obtained by Warner Bratzler method, which is considered as intermediate tenderness. The sausage was very well received by evaluators for taste. The TPA results of hardness, adhesiveness, springiness, cohesiveness, gumminess and chewiness were 2.01 $\mathrm{N}, j-8.53,0.830 .74,14.71 \mathrm{~N}$ and $12.26 \mathrm{~N}$ respectively, showing that spectacled caiman meat has good quality for the production of meat emulsions and, as the cooking loss percentage was $5.9 \%$, also has good stability.
\end{abstract}

Key words: Spectacled caiman meat, color, texture, sensory analysis.

\section{INTRODUCCIÓN}

La malnutrición y la subnutrición es un problema que afecta especialmente a los países subdesarrollados, esta enfermedad es tan grande que según las cifras presentadas por la Organización para la Agricultura y la Alimentación (FAO), en el periodo entre 2011 y 2013, en Colombia habían aproximadamente 5,1 millones de personas en estado de desnutrición, siendo las poblaciones pobres, especialmente en las zonas rurales, las más vulnerables a este problema donde por lo general el consumo de proteínas necesarios para el buen funcionamiento del cuerpo no se alcanza (1). Otro aspecto importante, es que actualmente las personas se preocupan mucho más por la composición de los alimentos que consumen, y en general buscan productos que contengan una buena fuente de proteínas y que además sean bajos en grasa y colesterol, esto hace que se busquen nuevas alternativas
Armando Alvis (1)

Pedro Romero (1)

Clemente Granados (2)

Miladys Torrenegra (3)

Nerlis Pajaro-Castro (4)

(1) Programa de Ingeniería de Alimentos. Grupo de Investigación Procesos y Agroindustria de Vegetales. Facultad de Ingenierías. Universidad de Córdoba. Córdoba, Colombia. (2) Programa de Ingeniería de Alimentos. Grupo de investigación INCAS (Ingeniería, Innovación, Calidad Alimentaria y salud). Facultad de Ingeniería. Universidad de Cartagena, Cartagena, Colombia. (3) Centrode Comercioy Servicios, Regional Bolívar. SENA. Grupodelnvestigación de Nano, Biotecnología e Innovación-GIBEI-. Cartagena, Colombia. (4) Programa de Medicina. Grupo de Ciencias Médicas y Farmacéuticas. Facultad de Ciencias de la Salud. Universidad de Sucre, Sincelejo, Sucre, Colombia.

Dirigir correspondencia a: Nerlis Pajaro-Castro

Programa de Medicina. Grupo de Ciencias Médicas y Farmacéuticas, Facultad de Ciencias de la Salud, Universidad de Sucre, Calle 16B No.13B-80, Sincelejo, Sucre, Colombia. Teléfono: (575)2818130

Email: nerlis.pajaro@unisucre.edu.co

Este trabajo fue recibido el 18 de Junio de 2016 y aceptado para ser publicado el 6 de Diciembre de 2016. que nos permitan suplir estas necesidades (2), aquí entran en juego las llamadas carnes exóticas las cuales cada día aumenta su consumo a nivel mundial. La carne de la babilla también conocido como caimán de anteojos (Caimán Crocodylus Fuscus), procedente de zoo-criaderos se presenta como una nueva alternativas que aparecen en el mercado, dado que es una carne que tiene un alto potencial nutricional, bajos niveles de grasa y alta proporción de ácidos grasos poli-insaturados a diferencia de los animales domésticos comúnmente utilizados para la alimentación humana (3). La carne de babilla es rica en proteínas y ácidos grasos poliinsaturados; con un sabor parecido a la carne de bagre lo que la hace muy apetecida en países como Tailandia, Australia, Alemania, Francia y gran parte de Asia, donde la carne de reptil es muy apetecida (4).

Colombia, según Andrés Valencia Pinzón, gerente general del ICA, está en capacidad de producir 150.000 kg de carne 
de babilla anualmente en los zoo-criaderos que funcionan de manera oficial en el país (5) estos centran su enfoque en la piel de la babilla dado a la importancia que representa para la industria marroquinera, empleando la carne de babilla principalmente como fuente de proteína para la alimentación de los animales más pequeños, como ingrediente de alimentos concentrados para animales, y en muchos casos, arrojada a las vertientes hídricas, lo que causa un gran deterioro del medio ambiente. Por lo anterior, es necesario establecer buenas prácticas de manufactura en el proceso de obtención de la carne a fin de garantizar su calidad final y poder aprovecharla de manera comercial e industrial (6).

El presente trabajo tuvo como objetivo, caracterizar la carne de babilla (Caimán Crocodylus Fuscus) en cautiverio, en cuanto a textura y color y elaborar una emulsión cárnica tipo salchicha y ver el grado de aceptabilidad.

\section{MATERIALES Y MÉTODOS}

Se emplearon 10 animales con un peso promedio de $15 \mathrm{~kg}$ obtenidos del zoo-parque los caimanes, ubicado en el municipio de Buenavista, Córdoba (Colombia). Se sacrificaron a través de una insensibilización con un golpe contundente en el cráneo, luego se realizó la extracción de la sangre, corte de la cabeza y se procedió a retirar la piel de manera manual. En el desuello se separó la piel del animal realizando incisiones alrededor del cuello y extremidades, y a lo largo del lomo desde la nuca hasta la cola aprovechando así la piel del vientre que resulta ser la más suave de todo el animal. Posteriormente se retiraron las reservas de grasa y por último la cola, descartando el resto de las partes. Las 10 colas de babilla, cada una de 2 $\mathrm{kg}$, fueron empacadas en cavas con Gel Pack para garantizar la cadena de frio hasta llegar a las instalaciones de la Universidad de Córdoba, se deshuesaron descartándose los huesos y empacando la carne al vacío para posteriormente almacenarla a una temperatura de $-20{ }^{\circ} \mathrm{C}$, para posteriormente hacer la caracterización respectiva.

\section{Color}

Para la determinación de color se utilizó el colorímetro Marca Huntelab ColorFlex EZ Spectrophotometer y se realizó la medición de los parámetros de color empleados en la meto- dología CIELAB, por medio de $L^{*}$ : luminosidad (negro- blanco), $a^{*}$ (verde -rojo), b* (azul-amarillo), método planteado por la Commission Internationale de l'Eclairage (CIE). Para esto se tomaron muestras en triplicado con un corte rectangular, aproximadamente $4 \mathrm{~cm}$ de largo por $2 \mathrm{~cm}$ de ancho y un espesor de $1 \mathrm{~cm}$, obtenidas de las colas de las babillas.

\section{Análisis de terneza}

Para el análisis de terneza de la carne de babilla se empleó un texturómetro marca TA-Tx plus Micro System Stable, utilizando la cuchilla Warner-Bratzler. Se utilizaron muestras rectangulares con medidas aproximadas a $8 \mathrm{~cm}$ de largo, 1 $\mathrm{cm}$ de ancho y $1 \mathrm{~cm}$ de espesor; obtenidas de colas de las babillas, estas se colocaron en el texturómetro, que usó 30 $\%$ de compresión, $1 \mathrm{~mm} /$ segundo de velocidad y una fuerza de 20 gf $(7,8)$.

Elaboración de salchicha de carne de babilla

Se realizó la formulación de la salchicha (tabla 1). El producto fue elaborado de la siguiente manera: Se inició con la molienda de la carne de babilla, la cual se utilizó semicongelada, posteriormente se buscó una reducción de tamaño más fino mediante el empleo del cutter, agregándole simultáneamente la sal nitrificada, a fin de liberar las proteínas miofibrilares, los fosfatos y todas las especias y condimentos (tabla 1). Luego se agregó la mitad del hielo y se continuó picando hasta afinar bien la carne, a una temperatura de 6 ${ }^{\circ} \mathrm{C}$. Se aumentó la velocidad del cutter a fin de obtener una pasta más homogénea. Se agregó la grasa de cerdo molida, posteriormente el almidón y el resto del hielo hasta llegar a una temperatura de 10 a $12{ }^{\circ} \mathrm{C}$. Seguidamente se procedió a embutir la salchicha, para lo cual se empleó tripa artificial en base a colágeno; se realizó el porcionado en unidades de 80 g aproximadamente, y finalmente, se continuó con el proceso de escaldado a una temperatura de $72{ }^{\circ} \mathrm{C}$, durante 40 minutos. Transcurrido este tiempo, se realizó un choque térmico a una temperatura de $4{ }^{\circ} \mathrm{C}$ a las salchichas y finalmente se llevó al horno para realizar el proceso de ahumado, para el cual se empleó un subproducto conocido como "aserrín", que facilita grandemente el manejo del fuego y confiere la posibilidad de maniobrar con el humo $(8,9)$.

\section{TABLA 1}

Formulación de la salchicha de carne de babilla.

$\begin{array}{cc}\text { Ingredientes } & \text { Cantidad (g) } \\ \text { Carne de babilla } & 3204 \\ \text { Sal nitrificada } & 74,0 \\ \text { Fosfato } & 16,02 \\ \text { Ajo en polvo } & 16,02 \\ \text { Cebolla en polvo } & 53,4 \\ \text { Condimento de salchicha } & 53,4 \\ \text { Comino } & 21,36 \\ \text { Nuez moscada } & 5,34 \\ \text { Realzador de sabor } & 5,34 \\ \text { Hielo } & 1368 \\ \text { Grasa de cerdo } & 462 \\ \text { Humo liquido } & 5,34 \\ \text { Almidón } & 106,8\end{array}$


Determinación de la estabilidad de la emulsión

Se obtuvo mediante el cálculo de pérdidas por cocción, para lo cual se tomó el peso de las salchichas una vez estuvieron moldeadas, siendo este el peso inicial; y se tomó como peso final, el peso de las salchichas una vez estuvieron escaldadas (9).

Análisis de perfil de textura (TPA)

Para el análisis de TPA de las salchichas de carne de babilla se empleó un texturómetro marca TA-Tx plus Micro System Stable. Se utilizaron muestras con medidas de $2 \mathrm{~cm}$ de largo y 2,5 cm de diámetro; estas se colocaron en el texturómetro, que usó $30 \%$ de compresión, $1 \mathrm{~mm} /$ segundo de velocidad y una fuerza de $20 \mathrm{gf}(9,10)$.

Análisis de color en la salchicha de carne de babilla

Para la determinación de color se utilizó el colorímetro marca Huntelab ColorFlex EZ Spectrophotometer y se realizó la medición de los parámetros de color empleados en la metodología CIELAB, por medio de L*: luminosidad (negro- blanco), $a^{*}$ (verde -rojo), b* (azul-amarillo). Se tomaron muestras con un corte circular, aproximadamente $4 \mathrm{~cm}$ de diámetro y un espesor de 0,5 cm. En todos los casos anteriores, las pruebas se realizaron a una temperatura ambiente $\left(25^{\circ} \mathrm{C}\right)$, tomando 3 réplicas de cada experimento (9).

\section{Análisis sensorial}

Para el análisis sensorial se utilizó una escala hedónica con las palabras me gusta mucho, me gusta moderadamente, ni me gusta ni me disgusta, me disgusta moderadamente, me disgusta mucho. Se escogieron 44 personas no entrenadas con el fin de evaluar el nivel de agrado de la salchicha de babilla (9).

\section{RESULTADOS}

Los parámetros de color CIELAB en la carne de babilla se presentan en la tabla 2 . El parámetro $L^{*}$ representa la luminosidad de color $\left(L^{*}=0\right.$ rendimientos negro y $L^{*}=100$ indica blanca), en este trabajo $L^{*}$ fue de $65,04 \pm 1,4$. La posición entre rojo y verde $\left(a^{*}\right.$, valores negativos indican verde mientras valores positivos indican rojo), este valor fue de $-0,98 \pm 0,63$; y su posición entre amarillo y azul ( $b^{*}$, valores negativos indican azul y valores positivos indican amarillo), este valor fue

TABLA 2

Parámetros de color CieLab de la carne de Babilla.

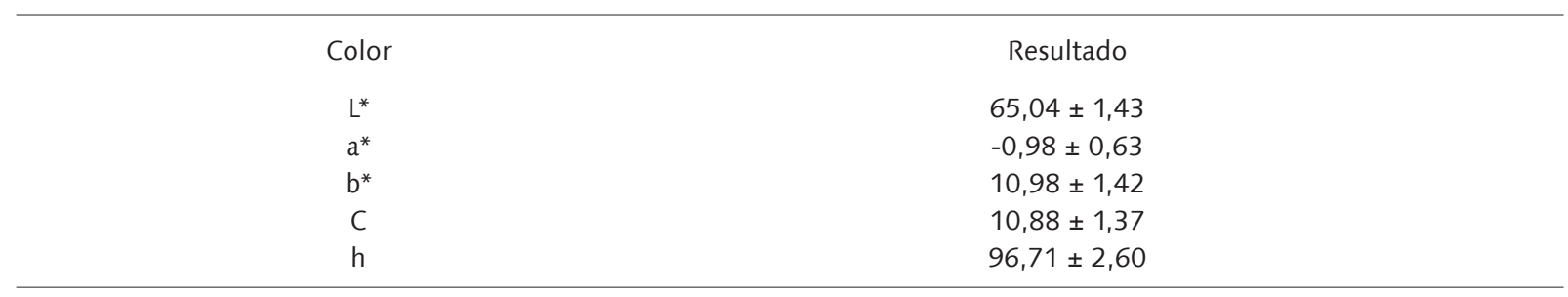

\section{FIGURA 1}

Resultado de terneza de la carne de babilla.

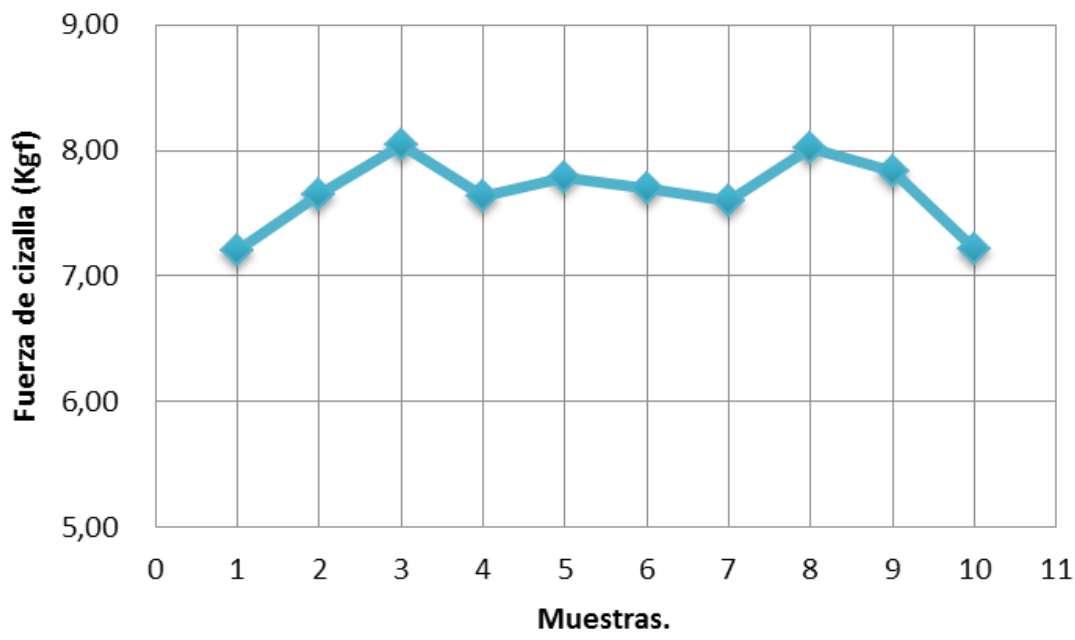


de $10,98 \pm 1,42$.

Los resultados de terneza de la carne de babilla se pueden observar en la figura 1, donde se reportan los datos de fuerza de cizalla para cada muestra en Kgf. De esta manera el valor promedio de fuerza de corte de la carne de babilla fue $7,67 \pm 0,27 \mathrm{Kgf}$. Con este resultado y de acuerdo a la clasificación de Warner Bratzler (FCWB), esta carne puede considerarse como una carne de terneza intermedia ya que el valor de fuerza de cizalla se encuentra en este rango $6<7,67<9,0 \mathrm{Kgf}(11)$.

Como se observa en la tabla 3, la estabilidad de la emulsión EE representada por la pérdida de jugo en cuanto al contenido acuoso fue de 5,89\%, Ramos et al., (12), así como Álvarez et al., (13) encontraron porcentajes de pérdida entre un 2 y $3 \%$ con carnes de vacuno y porcino respectivamente.

Los resultados del análisis de perfil de textura (TPA) en la salchicha (tabla 4), reflejan que el producto terminado presentó una menor dureza que la carne, esto se debe principalmente al procesado realizado, para el caso de la fracturabilidad este parámetro no arrojó ningún resultado ya que el producto durante las fases de compresión no alcanzó a fracturarse. En el caso de la adhesividad vemos que la salchicha no es adhesiva puesto que su valor resultó negativo, es decir, no se necesita realizar trabajo para separarse después de la compresión.

En la tabla 5 se observan los parámetros de color de la salchicha de carne de babilla.

De las 44 personas encuestadas en la escala hedónica para evaluar el agrado de la salchicha de babilla, 54,5\% expresaron que el producto les gustó mucho, mientras que $36,3 \%$ opinaron que la salchicha les gustaba moderadamente y 9,09\% expreso que ni le gustaba ni les disgustaba y ninguna optó por las opciones de me disgusta moderadamente y me disgusta mucho.

\section{DISCUSIÓN}

La carne de babilla presentó un valor del luminosidad $L^{*}=65,04$ por lo que puede ser considerada como blanca, ya que este valor nos da información de la coordenada negro/ blanco, es decir que posee una elevada claridad, lo cual podría deberse al mayor contenido de fibras blancas (14). Si se observa el valor de $a^{*}=-0,98 \pm 0,63$ el cual representa la coordenada verde/rojo, podemos notar que este se encuentra muy cercano al cero en el círculo cromático, sin embargo; éste parámetro es bastante elevado, esto lo podría estar presentando porque existe diferencia en las zonas donde se toman las muestras para el análisis y afecta los resultados, ya que la babilla es un animal de sangre fría por lo que sale a tomar el sol y esto hace que se afecte la coloración de la piel que está expuesta a este, el efecto en la fisiología del animal podría afectar la coloración de la carne en la zona donde se expone al sol (4), se puede notar que este tipo de carne se aleja del color rojo, por lo se puede inferir que el contenido de mioglobina es bajo, dado que esta proteína es la responsable en $90 \%$ del color rojo de la carne bovina.

En cuanto al parámetro $b^{*}=10,98$ se puede ver que está relativamente cerca del centro del diagrama cromático, sin embargo, se encuentra buscando hacia el color amarillo. Se puede observar en los valores de $L^{*}, a^{*} y b^{*}$, que no están tan

\section{TABLA 3}

Pérdidas por cocción en salchichas de babilla.

$\begin{array}{lccc}\text { Análisis } & \text { Peso inicial }(\mathrm{g}) & \text { Peso final }(\mathrm{g}) & \text { Pérdidas por Cocción (\%) } \\ \text { Estabilidad } & 3598 & 3386 & 5,89\end{array}$

\section{TABLA 4}

TPA de salchichas de diferentes animales.

\begin{tabular}{lcccc}
\hline Tipo de salchicha & Dureza & Elasticidad & Cohesividad & Gomosidad \\
Babilla & 2,01 & 0,83 & 0,74 & 14,71 \\
Atún* & 24,60 & 0,923 & 0,773 & 19,01 \\
Pavo** & 2,50 & 0,64 & 0,45 & ND \\
Pollo*** & 2,11 & 0,70 & 0,52 & ND \\
\hline Fuente: *Esta investigación ${ }^{* *}$ Granados y cols., $2013 .{ }^{* * *}$ Cortes y cols., 2010 & & & \\
\hline
\end{tabular}

\section{TABLA 5}

Parámetros de color de la salchicha de carne de babilla.

\section{Color}

$a^{*}$

$b^{*}$
Resultado

$73,47 \pm 0,04$

$1,85 \pm 0,32$

$13,46 \pm 0,17$ 
marcados hacia un lado o hacia el otro sino que más bien hacia el centro del circulo cromático, donde predominan los colores grises, eso se ve reflejado el valor de croma $C=10,88$ el cual describe lo llamativo o lo apagado de un color, tan cerca está el color ya sea al gris o al matiz puro, los colores en el centro son grises (apagados o sucios) y conforme avanzamos hacia la periferia se vuelven más saturados (vivos o limpios), el croma también se conoce como saturación.

Los valores de terneza de las carnes son muy variables de una especie a otra y entre la misma especie, debido a que están influidos por factores como la alimentación, la edad y el manejo del animal, pero pueden ser optimizados por el manejo que se le dé a la canal durante su faenado y almacenamiento, lo que mejora su calidad sensorial, es decir, que se produce una disminución de la resistencia al corte Vásquez y cols., (11) determinaron la fuerza de cizalla en carne de res $(5,17 \mathrm{Kgf})$, cordero (4,7 Kgf), emú (2,3 Kgf), avestruz (1,4 Kgf), ciervo (1,3 Kgf) y jabalí (2,3 Kgf) y comparándola con la obtenida en esta investigación se observó que la de babilla es más dura $(7,67$ Kgf) y muy similar en la textura a la carne de pollo (7,81 Kgf).

Comparando el TPA de la salchichas de babilla con salchichas de atún, pavo y pollo, se puede ver que las características de las primeras son muy similares a las reportadas por Cortes y cols., (15), para salchichas de pavo y pollo en cuanto a dureza y elasticidad difiere un poco de las de babilla en la cohesividad, en la cual es muy parecida a la reportada por Granados y cols., (16); si se observa la gomosidad de la salchicha de babilla es similar a la de la salchicha de atún, para este parámetro no se encontraron reportes en la salchichas de pavo y pollo tabla 4.

Se evidencia en la tabla 5 que la salchicha de babilla en comparación con la carne de babilla presentó un aumento

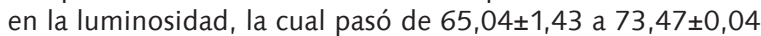
notándose en el producto terminado, el cual fue una salchicha muy blanca. El parámetro $a^{*}$ para el caso de la salchicha, presentó una leve inclinación hacia el rojo lo que se puede presentar por la adición de nitritos que les acentúa el color a los productos cárnicos. El valor de $b^{*}$ también presentó un pequeño aumento hacia la coordenada amarilla.

Joya y Bustacarra (2) elaboraron tres productos cárnicos (chorizo, longaniza y hamburguesa), con un $100 \%$ de carne de babilla y los evaluaron sensorialmente, los resultados de la encuesta de aceptación de los productos fueron satisfactorios ya que en términos generales fue de agrado. Alarcón y cols. (3) analizaron sensorialmente dos productos cárnicos (jamón de alta inyección y costillas ahumadas) elaborados con base en carne de babilla (color, textura, olor, sabor), se utilizaron consumidores no entrenados y se realizaron 400 evaluaciones para establecer el grado de aceptación. Estos productos presentaron altos niveles de aceptación (99\%) para las características de textura, sabor y olor a diferencia del color, lo cual se atribuyó al color pálido característico de la carne de babilla que contrasta con el color típico de los productos cárnicos de res y de cerdo, se asoció normalmente por el consumidor a productos cárnicos con atributos de frescura y salubridad.

De acuerdo con esto, podemos ver que las personas que aceptaron comer el producto en su mayoría les gustó mucho $(54,5 \%)$, por lo que puede existir un nicho de mercado al que se le pueden ofrecer este tipo de producto derivado de la carne de babilla.

\section{CONCLUSIONES}

La carne de babilla presenta unos parámetros de color en la escala CIELAB, que muestran que es bastante blanca y muy parecida a la carne de pollo y de pescado en lo relacionado con el color. Se considera como una carne de terneza intermedia ya que el valor de fuerza de cizalla se encuentra en este rango $6,0<7,67<9,0 \mathrm{Kgf}$, de acuerdo a la clasificación de Warner Bratzler (FCWB).

La emulsión tipo salchicha elaborada con carne de babilla presentó una buena estabilidad, ya que las pérdidas por cocción no alcanzaron el 6\%, y los parámetros TPA se encuentran dentro del rango normal para las salchichas. Presentó una buena aceptación ya que $65 \%$ de los encuestados les gustaría consumir el producto y en la prueba de escala hedónica tuvo muy buena aceptación en cuanto al sabor, ya que 54,5\% de los encuestados les gustó mucho el producto.

\section{RESUMEN}

En la presente investigación, se utilizaron 10 colas de babilla (Caiman crocodilus) de $2 \mathrm{Kg}$ cada una, empacadas al vacío a $-20{ }^{\circ} \mathrm{C}$ y almacenadas durante 8 días. Con la carne magra se elaboró una salchicha a la cual se le evaluó el color, la textura y la calidad sensorial para observar su frescura y a una salchicha elaborada análisis sensorial y un análisis de perfil de textura (TPA). Los parámetros de color fueron $L^{*}=65.04$, $a^{*}=-0.98$ y $b^{*}=0.98$, por lo que se considera un tipo de carne blanca lo cual está determinado por la alta luminosidad, se obtuvo una fuerza de corte con cizalla Warner Bratzler de 7,67 Kgf que se considera como de terneza intermedia. La salchicha elaborada presentó muy buena aceptación por parte de los evaluadores en cuanto a su sabor, en el TPA los resultados de dureza, adhesividad, elasticidad, cohesividad, gomosidad y masticabilidad fueron $2,01 \mathrm{~N},-8,53 \mathrm{j}, 0,83,0,74,14,71 \mathrm{~N} y$ $12,26 \mathrm{~N}$ respectivamente, evidenciando que la carne de babilla presenta una buena calidad para la elaboración de emulsiones cárnicas, además, presenta una buena estabilidad debido a que el porcentaje de pérdidas por cocción fue de 5,9\%.

Palabras clave: Carne de babilla, color, textura, análisis sensorial

Agradecimientos. Los autores agradecen a la Universidad de Cartagena, de Córdoba, de Sucre y el SENA por su apoyo en el desarrollo de este trabajo.

\section{REFERENCIAS}

1. FAO. 2013. The state of food insecurity in the world. http://www.fao.org/3/a-i4646s.pdf

2. Joya Fy Bustacara A. Elaboration of three meat products: Chorizo, Longaniza and Hamburguesa, with 100\% meat of Babilla. Thesis Zootecnista. University of the Salle. Bogotá, D.C. Colombia. 2007.

3. Alarcón M, López JH, Ortiz EV, Yepes DG, Mayorga WR. Sensory evaluation of meat meat products of babilla (Caimán crocodylus crocodilus). Rev Venezolana Ciencia Tecnol Alim. 2010; 1(2):184-92.

4. Quintana A. Sensory evaluation of alligator meat Moreletti. Tesis. Universidad de las américas Puebla. México. 2006.

5. Diario El Pais. Chinese "devour" meat of Colombian babilla. 2007. http://historico.elpais.com.co/historico/ mar232007/INT/babilla.html.

6. Castro F. y Gutiérrez Y. Design and assembly of a mobile station for slaughtering babilla (Caimán Crocodilus). Tesis Zootecnista. Universidad de la Salle. Bogotá, D.C. Colombia. 2009.

7. Granados C, Acevedo D, Cabeza A, Lozano A. Texture Profile Analysis in Bananas Pelipita, Hartón y Topocho. Inf Tecnol. 2014; 25(5):35-40.

8. Salas N, Lengua-Calle RL, Vásquez Becerra. Technological 
design of the processing of alpaca sausages of high protein content. Rev Industrial Data. 2014; 17(2):105-11

9. Hleap JI, González JM, Mora MF. Bromatological, sensory and microbiological evaluation of red tilapia sausages (Oreochromis sp.) with addition of earthworm flour (Eisenia Foetida). Vitae. 2012; 19(1):210-2

10. Hleap JI, Velasco VA. Analysis of texture properties during storage of sausages made from red tilapia (Oreochromis sp.). Fac Cs Agropecuarias. 2010; 8(2):46-56.

11. Vásquez E. Factors associated with meat quality. Part I: tenderness of beef in 40 livestock enterprises in the Caribbean region and the Middle Magdalena. Rev Corpoica. 2007; 8(2):60-5.

12. Álvarez D, Castillo M, Garrido MD, Bañón S, Nieto G, Díaz $P$, Payne FA. Effect of composition and processing time on the technological and optical properties of meat emulsions.
An Veterinaria Murcia. 2007; 23:25-34.

13. Ramos AG, Farias $M E$, Almada C, Crivaro N. Stability of Sausages with Hydrocolloids and Emulsifiers. Información Tecnol. 2004; 15(4):91-4.

14. Paredes D. Characterization of wild boar (Sus scrofa) from animals raised in Chile. Tesis Ing. Alim. Universidad Austral de Chile. Valdivia Chile. 2002.

15. Cortés M, Valera E, Meza M, Alfaro R, Güemes N, Hernández $J F$, Soto $S$. Texture of sausages made with mixtures of turkey and chicken meat. XII Congreso nacional de ciencia y tecnología de alimentos. Universidad de Guanajuato. México. 2010.

16. Granados C, Guzmán L, Acevedo D. Proximal, sensory and texture analysis of sausages made with by-products from the tuna processing industry (Scombridae thunnus). Inf Tecnol. 2013; 24(6):29-34. 